\title{
Guest editorial: special issue on high mobility wireless communications
}

\author{
Pingzhi FAN ${ }^{1 *}$, Erdal PANAYIRCI ${ }^{2}$, Ping $\mathrm{LI}^{3}$, Chengxiang $\mathrm{WANG}^{4}$, Vahid TAROKH \\ 1. School of Information Science and Technology, Southwest Jiaotong University, Chengdu 610031, China \\ 2. Dept of Electrical and Electronics Engineering, Kadir Has University, 34083, Cibali, Istanbul, Turkey \\ 3. Dept of Electronic Engineering, City University of Hongkong, Kowloon, Hong Kong SAR \\ 4. School of Engineering \& Physical Sciences, Heriot-Watt University, Edinburgh EH14 4AS, UK \\ 5. School of Engineering and Applied Sciences, Harvard University, Cambridge, MA 02138, USA
}

Key words: high mobility; wireless communications; high speed trains (HSTs)

(C) 2012 JMT. All rights reserved.

$\mathrm{T}$ he high-speed railway and high-way networks are now expanding at a phenomenal speed in China and in many other parts of the world. The related broadband wireless communication over high-speed trains and highway vehicles is a very challenging task due to hostile transmission channel conditions. The demand for such services is growing rapidly, following the proliferation of laptop/tablet computers and smart phones. This motivates the research on wireless communications in the high mobility environments.

This special issue aims at putting together the new achievements and developments in this field. There are 11 papers in this special issue, which have been organized into three thematic groups. The first group of 4 papers deals with the rapidly time-varying channel measurement, modeling and estimation. The second group of 3 papers addresses the Doppler effects and signal detection. The last group of 4 papers contributes to the relays, networking coding, and the fast handover schemes for high mobility scenarios.

Among the four papers dealing with rapidly timevarying channel modeling and estimation, two papers are concerned with channel measurement and modeling, namely, "High-speed railway channel measurements and characterizations: a review" (Tao ZHOU, et al.), and "Propagation characteristics of the wideband high-speed railway channel in viaduct scenario at $2.35 \mathrm{GHz}$ " (Yunling GUO, et al.). The former provides an overview

Received Dec. 18, 2012; revision accepted Dec. 20, 2012 ${ }^{*}$ Corresponding author. Tel.: +86-28-87600740, Fax:+86-2887600743, E-mail: pingzhifan@gmail.com

(C) 2012 JMT. All rights reserved doi: 10.3969/j.issn.2095-087X.2012.04.001 of the recent channel measurement campaigns in highspeed railways (HSRs), along with some specific measurement and modeling results. The later presents a channel measurement and modeling in a railway viaduct scenario between Zhengzhou and Xi'an, a passenger dedicated line, with a bandwidth of $50 \mathrm{MHz}$ at $2.35 \mathrm{GHz}$. The third paper, "Antenna calibration using channel prediction for time-varying channels" (Yamin SONG, et al.), proposes an antenna calibration method for rapidly time-varying channels. It is shown that the proposed method can well compensate the performance loss and significantly improve the antenna calibration performance for time-varying channels. The fourth paper, "Modified clustered comb pilot-aided fast timevarying channel estimation for OFDM system" (Xin LI, et al.), proposes a modified clustered comb pilot-aided structure with improved channel estimation performance, where the time varying channel is approximated by a basis expansion model (BEM). Based on complexexponential BEM (CE-BEM) model, a suboptimal-pilot structure is also proposed.

Concerning the three papers related to the topic of Doppler effects and signal detection, the first paper, "Doppler frequency offsets estimation and diversity reception scheme of high-speed railway with multiple antennas on separated carriage" (Yaoqing YANG, et al.), presents a joint Doppler frequency offset (DFO) estimation and channel estimation algorithm, together with a theoretical Crammer Rao bound (CRB), based on Ricean channel model, to exploit the cooperative multiantenna diversity gains. In the paper "Signal detection through circular convolution reconstruction for OFDM system in fast varying channel" (Hai ZUO, et al.), a signal detection algorithm is devised for the orthogonal 
frequency division multiplexing (OFDM) system in the presence of fast time-varying channel which is represented by a piece-wise linear variant model with normalized Doppler frequency of less than 0.2. It is shown that the proposed method can not only track the channel variation, but also promise better performance gain in the OFDM symbol detection. The final paper of this group, "Achievable secrecy rate of bit-interleaved coded modulation schemes" (Weichen XIANG, et al.), studies the impact of various modulation mapping strategies and signal constellation shapes on the secrecy rates achievable with bit-interleaved coded modulation (BICM) schemes. It is concluded that proper design of signal mapping can significantly enhance the achievable secrecy rate in BICM schemes.

The four remaining papers deal with topics related to the relays, networking coding, and the fast handover schemes for high mobility wireless communications. The paper "asymptotic analysis of multi-branch amplify-and-forward two-way relaying in Nakagami- $m$ fading with arbitrary fading parameter" (Jing YANG, et al.) investigates the asymptotic performance for multibranch dual-hop two-way amplify-and-forward (AF) relaying networks in independently but not necessarily identically distributed Nakagami- $m$ fading channels with arbitrary parameter $m \geq 0.5$. Utilizing the obtained result, asymptotic outage probability expression is derived, from which the diversity order and coding gain are analyzed. By considering jointly the wireless relay and network coding, the paper "Optimal power allocation for complex field network coding scheme with the $K$-th best relay selection" (Xi CAI, et al.) proposes a complex field network coding (CFNC) scheme and an optimal power allocation algorithm, where the $K$-th best relay is selected to forward the multiplexed signal to the destination. The subsequent paper "Network coding with diversity and outdated channel state information" (Tong CHEN, et al.) applies network coding with diversity (NCD) and outdated channel state information (CSI) to achieve a form of selection diversity, and extend NCD to cooperative multiple access channels. To handle the serious high mobility management problem, the last paper, "Positionassisted fast handover schemes for LTE-advanced network under high mobility scenarios" (Ming FEI, et al.), proposes two position-assisted fast handover schemes $(A$ and $B)$ for LTE-A system under very high mobility scenarios. System level simulation shows that, Scheme $A$ could reduce inter-site handover delay by about $50 \mathrm{~ms}$, while Scheme $B$ could cut down nearly $50 \%$ of all handovers when the time to trigger (TTT) is $0 \mathrm{~ms}$. Besides, as TTT gets larger, Scheme B has much better success rate.

\section{Acknowledgements}

The guest editors would like to thank the authors of all submitted papers for considering this special issue to disseminate their work and all the reviewers for their conscientious work. Our special thanks also go to Dr. Qingchun CHEN for his excellent assistance in making this special issue possible. This work was partially supported by the Major State Basic Research Development Program of China (973 Program No. 2012CB316100), the National Natural Science Foundation of China (NSFC No. 61032002), and the Innovative Intelligence Base Project (111 Project No. 111-2-14).

(Editor: Junsi LAN) 